Disclosure. We would like to thank Mr. Hiroyuki Watanabe for his assistance in English language editing.

Financial support: none. Conflict of interest: none.

${ }^{1}$ Saravio Central Institute, Saravio Cosmetics Ltd., 1356-6 Oaza Tsurumi, Beppu, Oita, 874-0840 Japan ${ }^{2}$ Department of Pathophysiology and Therapeutics of Diabetic Vascular Complications,

Kurume University, Fukuoka, Japan

${ }^{3}$ Department of Matrix Medicine, Oita University, Yufu, Oita, Japan $<$ kaseda@saravio.jp>

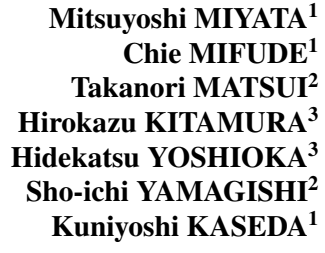

1. Ott C, Jacobs K, Haucke E, Navarrete Santos A, Grune T, Simm A. Role of advanced glycation end products in cellular signaling. Redox Biol 2014; 2: 411-29.

2. Fan $X$, Sell DR, Zhang J, et al. Anaerobic vs aerobic pathways of carbonyl and oxidant stress in human lens and skin during aging and in diabetes: A comparative analysis. Free Radic Biol Med 2010; 49: 847-56.

3. Pageon $\mathrm{H}$. Reaction of glycation and human skin: the effects on the skin and its components, reconstructed skin as a model. Pathol Biol (Paris) 2010; 58: 226-31

4. Botchkarev VA, Kishimoto J. Molecular control of epithelialmesenchymal interactions during hair follicle cycling. J Investig Dermatol Symp Proc 2003; 8: 46-55.

5. Invi S, Itami S. Molecular basis of androgenetic alopecia: From androgen to paracrine mediators through dermal papilla. J Dermatol Sci $2011 ; 61: 1-6$.

6. Kwack MH, Ahn JS, Kim MK, Kim JC, Sung YK. Dihydrotestosteroneinducible IL-6 inhibits elongation of human hair shafts by suppressing matrix cell proliferation and promotes regression of hair follicles in mice. J Invest Dermatol 2012;132: 43-9.

7. Trüeb RM, Tobin DJ. Aging Hair, 1st edn. Berlin: Springer-Verlag, 2010.

\section{Methotrexate-associated lymphoprolifera- tive disorder: Sequential development of angioimmunoblastic T-cell lymphoma-like lymphoproliferation in the lymph nodes and diffuse large $B$-cell lymphoma in the skin in the same patient}

A 66-year old Japanese woman was diagnosed with polymyalgia rheumatica in October 2012 and was treated with methotrexate (MTX) at a dose of $10 \mathrm{mg} /$ week. Swelling of the inguinal lymph nodes appeared in February 2013. Histological examination of the lymph nodes revealed numerous, medium-to-large, atypical mononuclear cells with irregular nuclei (figure 1A). Increased numbers of $\mathrm{CD} 21^{+}$follicular dendritic cells were observed around the high endothelial venules (figure $1 B$ ). The neoplastic cells stained positively for CD3 and CD5 but not for CD20 and bcl-6. Epstein-Barr virus (EBV)-encoded RNA (EBER) positive cells by in situ hybridization were also positive for $\mathrm{CD} 20$ and $\mathrm{CD} 79 \alpha$, but not $\mathrm{CD} 3$ (figure $1 C$ ).
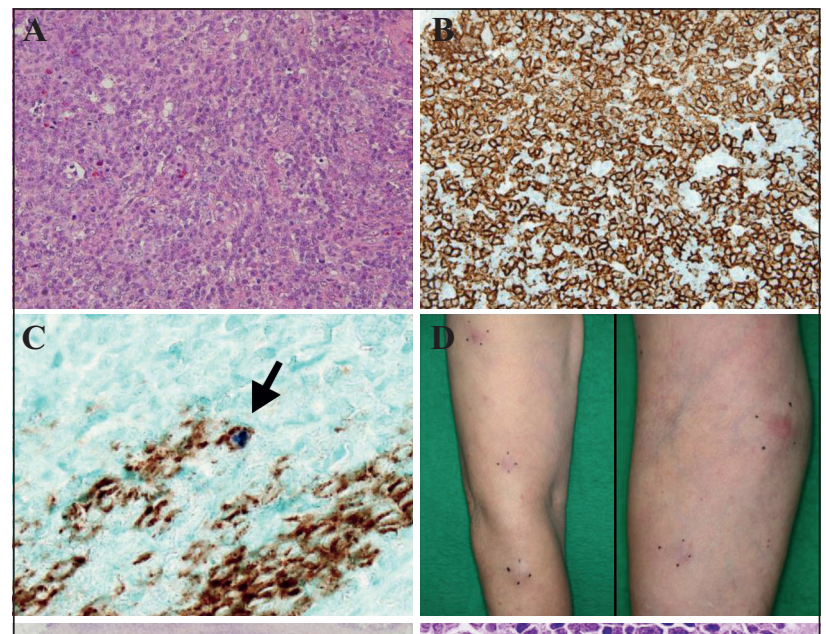

E

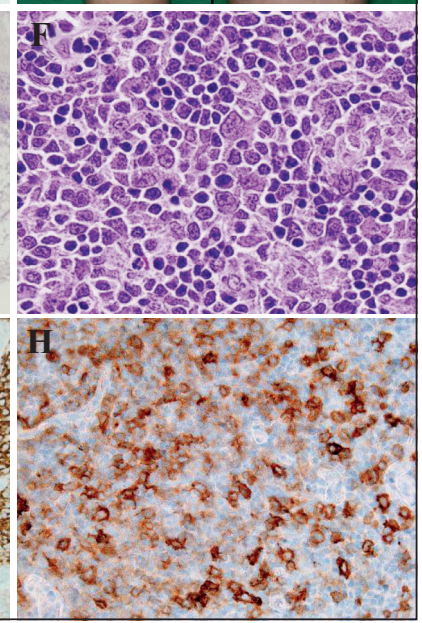

Figure 1. (A) Histological examination of lymph nodes. Numerous, medium-to-large sized, atypical, mononuclear cells with irregular nuclei (Hematoxylin-eosin, $\times 400)$. (B) Increasing number of $\mathrm{CD} 21^{+}$follicular dendritic cells around high endothelial venules $(\times 400)$. (C) In situ hybridization for Epstein-Barr virus (EBV)-encoded RNA (EBER), followed by the immunostaining of $\mathrm{CD} 20$. $\mathrm{EBER}^{+}$cells were positive for CD20 (indicated by arrow) $(\times 800)$. (D) Multiple subcutaneous nodules in her bilateral upper extremities. (E, F) Histopathological examination of the nodule on the arm. Diffuse infiltration of medium-to-large sized, atypical lymphocytes in the subcutaneous tissues (Hematoxylin-eosin, (E) $\times 100,(\mathbf{F}) \times 800)$. $(\mathbf{G}, \mathbf{H})$ Atypical lymphocytes were positive for CD20 (G) and CD30 (H) $(\times 400)$.

Quantitative polymerase chain reaction for plasma EBV DNA showed 290 copies $/ 10^{6}$ cells (normal: $<20$ ). The diagnosis of MTX-associated angioimmunoblastic Tcell lymphoma (AITL)-like lymphoproliferative disorders (LPD) was established and MTX was withdrawn in February 2013. Immunosuppressive agents, such as cyclosporine, tacrolimus and corticosteroids, were not used after the withdrawal of MTX. Swelling of the lymph nodes completely disappeared 6 months after the discontinuation of MTX. In April 2014, she noticed multiple 2-to-3 $\mathrm{cm}$ subcutaneous nodules in her bilateral upper extremities (figure 1D). Abnormal laboratory results included only elevated soluble interleukin 2 receptor $(1291 \mathrm{U} / \mathrm{ml}$, 
normal: 135-483). Histopathological examination of the nodule revealed diffuse infiltration of medium-to-large polymorphic atypical lymphocytes in the subcutaneous tissues (figures $1 E, F$ ). There were no Reed-Sternberg cells. The atypical lymphocytes were positive for CD20 (figure $1 G$ ), CD30 (figure $1 H$ ), CD10 and bcl-6 but not CD3 and CD5. Fifty percent of atypical cells were positive for MIB-1. EBER positive lymphocytes were detected by in situ hybridization. The diagnosis of MTX-LPD in the skin, comprised of a diffuse large B-cell lymphoma (DLBCL), was established. During a four-month follow-up period, the cutaneous nodules spontaneously regressed.

MTX has an immunosuppressive effect and is thought to be associated with the development of LPD. MTX-associated LPD is classified as "other iatrogenic immunodeficiencyassociated LPD" in the World Health Organization classification of tumors of hematopietic and lymphoid tissues [1]. 48 MTX-LPD patients treated with MTX until the diagnosis of LPD for periods of 2 to 131 (median 54) months have been reported [2]. The spontaneous regression of LPD following the withdrawal of MTX strongly suggests the potential pathogenetic role of MTX in LPD. However, the pathogenesis of the development of LPD by MTX has not been clearly established. Patients with RA develop LPD at a frequency 2 to 5.5 times higher than the general population [3], suggesting that the abnormal immune state of RA might also contribute to the development of LPD. In our case, it is possible that a polymyalgia rheumatica-induced abnormal immune state might also have been associated with the pathogenesis of LPD. Further studies are required to clarify the association of MTX with the development of LPD.

Histologically, DLBCL (60.4\%) and Hodgkin lymphoma (12.5\%) are common in MTX-LPD [2]. Approximately half of all MTX-LPD develop in extranodal sites, such as the lung, kidney, gastrointestinal tract and bone marrow [2]. However, reports of cases of MTX-LPD developing in the skin are limited [4]. The present case is rare in that the BCL in the skin developed after the cessation of MTX and two types of LPD, i.e., AITL-like and BCL, developed sequentially. Kikuchi et al. also reported a patient with TCL followed by BCL and suggested that long-term chemotherapy and EBV infection might be associated with the development of multiple lymphomas [5].

EBV is detected at high frequency in MTX-LPD (27.1\%; 13/48) [2]. Spontaneous remissions of EBV-LPD are common, especially when there is ulceration [6]. The five-year survival rate of EBV-positive MTX-LPD (76.3\%) is better than that of EBV-negative (54\%) [2]. Rizzi et al. reported that MTX-LPD patients who achieved complete remission (CR) exhibited increased frequencies of DLBCL (24\%), extranodal involvement (32\%) and EBV infection (56\%), and that near CR occurred within four weeks after the discontinuation of MTX [7]. Our case fulfilled all these favorable conditions for CR, suggesting that our patient required a period of a careful wait-and-see approach, rather than more aggressive cytotoxic therapies. Widespread use of immunosuppressive agents will lead to an increase in the frequency of LPDs. It is important for dermatologists to recognize the diagnosis and appropriate treatment of MTX-LPD.
Disclosure. Financial support: none. Conflict of interest: none.
Department of Dermatology,

Gunma University Graduate School

of Medicine,

3-39-22 Showa, Maebashi,

Gunma 371-8511, Japan

<smotegi@gunma-u.ac.jp>

\section{Hirohisa ISHIBUCHI Sei-ichiro MOTEGI Masayoshi YAMANAKA Hiroo AMANO Osamu ISHIKAWA}

1. Gaulard P, Swerdlow SH, Harris NL, Jaffe ES, Sundström C. Other iatrogenic immunodeficiency-associated lymphoproliferative disorders. In: Swerdlow SH, Campo E, Harris NL et al. eds. World Health Organization Classification of Tumours of Haematopoietic and Lymphoid Tissues. Lyon: IARC Press, 2008: 350-1.

2. Thomas E, Brewster DH, Black RJ, Macfarlane GJ. Risk of malignancy among patients with rheumatic conditions. Int J Cancer 2000; 88: 497-502.

3. Hoshida $Y, X u J X$, Fujita $S$, et al. Lymphoproliferative disorders in rheumatoid arthritis: clinicopathological analysis of 76 cases in relation to methotrexate medication. J Rheumatol 2007; 34:322-31.

4. Koens L, Senff NJ, Vermeer $M H$, Willemze $R$, Jansen PM. Methotrexate-associated B-cell lymphoproliferative disorders presenting in the skin: A clinicopathologic and immunophenotypical study of 10 cases. Am J Surg Pathol 2014; 38: 999-1006.

5. Kikuchi A, Anzai H, Kosuge H, Naka W, Nishikawa T. Aggressive B-cell lymphoma induced by Epstein-Barr virus infection in erythrodermic cutaneous T-cell lymphoma. Br J Dermatol 1997; 136: 124-8.

6. Dojinov $S D$, Venkataraman $G$, Raffeld $M$, Pittaluga $S$, Jaffe ES. EBV positive mucocutaneous ulcer-a study of 26 cases associated with various sources of immunosuppression. Am J Surg Pathol 2010; 34: 405-17.

7. Rizzi R, Curci $P$, Delia $M$, et al. Spontaneous remission of "methotrexate-associated lymphoproliferative disorders" after discontinuation of immunosuppressive treatment for autoimmune disease. Review of the literature. Med Oncol 2009; 26: 1-9.

doi: $10.1684 /$ ejd.2015.2582

\section{Dermoscopic findings of piloleiomyoma with hypertrichosis}

Cutaneous leiomyomas are benign tumors arising from smooth muscle cells. Herein, we describe a rare case of piloleiomyoma with hypertrichosis, in which the dermoscopic examination revealed features similar to those of dermatofibroma.

A 51-year-old man presented to our hospital with a nodule on his shoulder, which he had noticed 1.5 months earlier. Physical examination revealed a firm, brownish, painful nodule, $12 \mathrm{~mm}$ in diameter, with hypertrichosis on the left shoulder (figure 1A). The dermoscopic findings showed a central white patch, a delicate pigment network, several dotted vessels and hypertrichosis (figure $1 B$ ). His family history and medical history were unremarkable. Histopathological examination revealed a slightly thickened epidermis with parakeratosis and basal pigmentation, and a poorly demarcated eosinophilic tumor located within the dermis. The tumor consisted of interlacing bundles of spindle cells with no sign of atypism, intermingled with various amounts of collagen bundles; a hair follicle was 